\title{
Relecture mécanologique de l'histoire des télescopes
}

Mechanological reinterpretation of the history of telescopes

\section{Thomas Guy et Vincent Bontems}

\section{(2) OpenEdition \\ 1 Journals}

Édition électronique

URL : http://journals.openedition.org/artefact/3981

DOI : 10.4000 /artefact.3981

ISSN : 2606-9245

Éditeur :

Association Artefact. Techniques histoire et sciences humaines, Presses universitaires du Midi

\section{Édition imprimée}

Date de publication : 15 octobre 2019

Pagination : 99-118

ISBN : 978-2-8107-0650-1

ISSN : 2273-0753

\section{Référence électronique}

Thomas Guy et Vincent Bontems, «Relecture mécanologique de l'histoire des télescopes », Artefact [En ligne], 10 | 2019, mis en ligne le 06 août 2020, consulté le 28 novembre 2020. URL : http:// journals.openedition.org/artefact/3981; DOI : https://doi.org/10.4000/artefact.3981

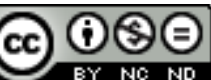

Artefact, Techniques, histoire et sciences humaines est mise à disposition selon les termes de la Licence Creative Commons Attribution - Pas d'Utilisation Commerciale - Pas de Modification 4.0 International. 


\section{Relecture mécanologique de l'histoire des télescopes}

\section{Thomas Guy et Vincent Bontems}

\section{Résumé}

Cet article propose de retracer l'histoire des télescopes en adoptant le point de vue de la mécanologie génétique. Cette approche en histoire des techniques vise à dépasser les oppositions qui existent entre les écoles internalistes et externalistes en centrant l'analyse sur les processus d'évolution technique plutôt que sur l'objet ou le système technique. Moins sensible aux surdéterminations socioculturelles pesant sur les objets du quotidien et s'étendant sur plus de quatre siècles, l'histoire des télescopes nous est apparue comme un choix judicieux pour tester la pertinence de notre méthode. En analysant les processus de conception, ainsi que la compétition entre les différentes lignées de télescopes, nous espérons parvenir à une meilleure compréhension des processus d'évolution technique sous-tendant le développement de ces instruments.

\section{Mots-clés}

mécanologie génétique, lignées techniques, télescopes, Simondon, TRIZ, contradictions techniques

99 Thomas Guy et Vincent Bontems, « Relecture mécanologique de l'histoire des télescopes », Artefact, 10, 2019, p. 99-118. 


\section{Mechanological reinterpretation of the history of telescopes}

\section{Abstract}

This article traces the history of telescopes by adopting the point of view of genetic mecanology. This approach, in the field of history of technology, aims to overcome the existing oppositions between internalist and externalist schools by focusing the analysis on the evolutionary technical processes rather than on the object or the technical system. Less sensitive to socio-cultural overdeterminations weighing on everyday objects and extending over more than four centuries, the history of telescopes appeared as a judicious choice to test the relevance of our method. Analysing the design processes as well as the competition between the different lineages of telescopes, we hope to achieve a better understanding of the technical evolution processes underpinning the development of these instruments.

genetic mechanology, technical lineages, telescopes, Simondon, TRIZ, technical contradictions 


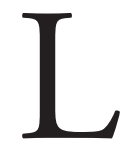

es historiens de l'astronomie ont coutume de distinguer l'astronomie prétélescopique de l'astronomie telle que nous la connaissons aujourd'hui ${ }^{1}$. L'invention de la lunette de Galilée en 1609, tenue pour la première occurrence du télescope dans l'histoire, a constitué un profond bouleversement des pratiques astronomiques : désormais les astronomes possédaient un instrument permettant d'acquérir des informations à propos d'objets extrêmement lointains, et dont la taille dépasse largement l'échelle humaine. La lunette astronomique a révolutionné l'astronomie par l'importance et la précision des observations réalisables, mais aussi parce qu'elle opéra un premier déphasage au sein des échelles d'observation $^{2}$. À la modification des pratiques scientifiques s'ajoutait une modification de la vision que l'Homme avait de sa place dans le $\operatorname{cosmos}^{3}$; ce changement épistémologique et ontologique constituant une révolution aussi bien scientifique $e^{4}$ quastronomique $^{5}$. Si des instruments d'observation existaient depuis longtemps - astrolabes, sphères armillaires et autres quadrants -, ceux-ci ne permettaient pas de dépasser les possibilités perceptives humaines alors que le télescope, en tant que dispositif amplificateur, allait devenir l'instrument central de l'astronomie. L'apparition de la lunette en 1609 signe le triomphe de la technicisation des observations et ouvre la voie aux autres champs scientifiques : "jusqu'aux alentours de 1600, l'astronomie était l'unique science utilisant des instruments. En 1700, aucune science ne pouvait prétendre s'en passer $^{6} \%$.

Mais des premières lunettes aux grands observatoires modernes, les dispositifs techniques mobilisés en astronomie ont énormément évolué. Nous proposons de retracer cette histoire selon le point de vue de la mécanologie génétique. Cette méthode d'analyse des évolutions des lignées techniques prolonge le projet mécanologique de Jacques Lafitte ${ }^{7}$ en l'enrichissant de la méthode génétique de Gilbert Simondon ${ }^{8}$. Il s'agit en particulier de

\footnotetext{
1. Walker, 1999, p. 12.

2. Simondon, 1961, p. 237.

3. Koyré, 1962.

4. Khun, 2008.

5. Koyré, 2016.

6. Séris, 2013, p. 208.

7. Lafitte, 1932, p. 68 : «La science des machines, ou mécanologie, science normative, n’a d'autre but que l'étude et l'explication des différences qui s'observent entre les machines ".

8. Simondon, 2012, p. 23 : « comme dans une lignée phylogénétique, un stade défini d'évolution contient en lui des structures et des schèmes dynamiques qui sont au principe d'une évolution des
} 
repérer l'apparition, l'évolution et la diffusion des différents schèmes techniques afin de retracer les différentes lignées de la famille des télescopes. Reprenant les catégories de Simondon, le télescope, en tant que réalisation effective d'un schème technique particulier dans un milieu associé, sera qualifié d'individu technique. Ses composants, indifférents à la nature du milieu seront qualifiés d'éléments et les dispositifs englobants, et articulant plusieurs milieux, d'ensembles techniques. Si le concept de lignées techniques nous semble être l'outil méthodologique le plus à même de proposer une reconstruction synthétique et robuste de l'histoire des télescopes c'est parce qu'il rattache l'évolution de ces dispositifs techniques à une dynamique - à une genèse ${ }^{9}$ pour reprendre le vocabulaire simondonien - d'évolution, et évite ainsi l'écueil d'une classification purement utilitariste des objets techniques.

En effet, selon l'étymologie, un télescope est ce qui permet de "voir loin » $(\tau \eta \lambda \varepsilon \sigma \kappa о \pi \omega)$, mais, du point de vue mécanologique, nous appellerons " télescope » tout dispositif technique permettant de collecter et de focaliser un rayonnement électromagnétique. Cela correspond à un nombre limité de schèmes techniques. Encore aujourd'hui, on ne connaît que deux principes réalisant cette double opération : il s'agit des phénomènes de réfraction lumineuse (à l'œuvre dans les lentilles) et de réflexion (à la surface de miroir notamment). Ces deux principes physiques se traduisent en deux schèmes techniques à l'origine des deux lignées de télescopes : les télescopes réflecteurs, ou simplement télescopes en français et les télescopes réfracteurs, ou lunettes ${ }^{10}$. Historiquement, c'est ce dernier, basé sur le principe de réfraction qui donna naissance au premier instrument permettant d'observer les astres en collectant et focalisant leur rayonnement.

\section{Vie et mort des télescopes réfracteurs}

Nous ferons débuter l'histoire du télescope en 1609, année à laquelle Thomas Harriot réalise les premiers dessins de la surface lunaire en utilisant une lunette (dutch trunke $\left.{ }^{11}\right)$, quelques mois avant que Galilée ne

\footnotetext{
formes ".

9. Simondon, 2012, p. 22.

10. On trouve aussi, mais plus rarement, les dénominations de " télescopes catoptriques " et de " télescopes dioptriques".

11. King, 2011, p. 33.
} 
l'utilise à son tour pour observer le mouvement des astres ${ }^{12}$. Si les pouvoirs grossissants des lentilles étaient connus depuis plusieurs siècles ${ }^{13}$, l'idée d'associer une lentille convexe et une lentille concave au sein d'un même dispositif afin d'obtenir un instrument permettant d'observer des objets éloignés ne fut formulée qu'en 1586, dans l'ouvrage Magia Naturalis de l'Italien Giambattista della Porta. La paternité de cette invention sera néanmoins disputée entre plusieurs opticiens hollandais. Entre septembre et octobre 1608, Hans Lippershey, Jacob Metius et Zacharias Janssen déposeront chacun une demande de brevet pour " un instrument permettant de voir les choses lointaines comme si elles étaient proches ${ }^{14}$ ", bientôt appelé « lunette d'approche ». Dotée d'un facteur de grossissement de seulement trois et possédant d'importantes d'aberrations, optiques comme chromatiques, la lunette d'approche est considérée à ses débuts comme un objet anecdotique ${ }^{15}$.

Mais du fait de son perfectionnement, puis de son adoption généralisée par la marine, notamment militaire, qui voit dans cet objet technique un formidable instrument de navigation, la lunette d'approche va rapidement se propager dans toute l'Europe jusqu'à parvenir dans les mains du mathématicien et astronome italien Galilée ${ }^{16}$. Si son nom est aujourd'hui associé à l'invention de la lunette astronomique, c'est parce qu'il est l'un des premiers à saisir le potentiel scientifique d'un tel dispositif, et à voir en lui le moyen de faire entrer l'astronomie dans une nouvelle ère ${ }^{17}$. Instrumentaliste brillant, il perfectionne la lunette hollandaise, diminuant les aberrations latérales et allant jusqu'à obtenir un facteur de grossissement de 20, puis de 30, lui permettant de réaliser, entre autres, les premières observations de Jupiter et de ses satellites.

Il est à noter que la lunette d'approche, devenue lunette astronomique est l'exemple même d'une " technique sans science », c'est-à-dire d'une invention majeure qui ne découle pas de l'application d'un principe scientifique qui l'aurait précédé : dès les premières lignes de la Dioptrique (Discours

12. Galileo Galilei, 1992 (1 ${ }^{\text {re }}$ éd. 1610).

13. Albert Van Helden, 977, p. 1-67.

14. Van Helden, Dupré, van Gent, Zuidervaart, 2010.

15. Couderc, 1982, p. 95.

16. King, 2011, p. 34.

17. Le premier utilisateur de la lunette à des fins astronomiques est Thomas Harriot, mathématicien et astronome anglais qui, quatre mois avant Galilée, l'utilise pour observer la surface de la Lune. 
Premier), Descartes nous parle ainsi de " ces merveilleuses lunettes qui, n'étant en usage que depuis peu, nous ont déjà découvert de nouveaux astres dans le ciel, et d'autres nouveaux objets dessus la Terre, en plus grand nombre que ne sont ceux que nous y avions vu auparavant ", et d'ajouter "Mais, à la honte de nos sciences, cette invention, si utile et si admirable, n'a premièrement été trouvée que par l'expérience et la fortune ${ }^{18}$.»

La lunette de Galilée va se diffuser rapidement et subir nombre d'améliorations, notamment par Kepler qui, en 1611, propose dans son ouvrage Dioptricae une étude mathématique de la théorie des dioptres et une nouvelle architecture pour la lunette ${ }^{19}$. Désormais composée de deux lentilles convexes et d'un tube non plus télescopique mais fixe, la lunette de Kepler permet d'atteindre des grossissements plus importants au prix d'un allongement du tube et d'une image retournée. Dans la mesure où l'observation des astres lointains ne dépend pas d'une orientation préférentielle, ce dernier point est peu problématique - au contraire de l'allongement du dispositif qui va, on le verra par la suite, enclencher une dynamique plus problématique.

104 Aussi différents soient-ils, les types galiléen et képlérien de lunettes présentent le même inconvénient de recourir à des lentilles et donc de présenter des aberrations chromatiques ${ }^{20}$. Celles-ci sont dues à la variation de l'indice de réfraction du verre composant les lentilles qui dépend de la longueur d'onde de la lumière qui les traverse : toutes les couleurs du spectre ne sont pas déviées avec la même intensité et sont donc focalisées à des distances différentes ${ }^{21}$. Si, par exemple, la mise au point est effectuée pour le rouge, le bleu est alors flou et inversement : on note alors la présence d'irisations qui nuisent à la qualité de l'image en sortie du système optique. La suppression de ces aberrations chromatiques a constitué le premier grand défi de l'instrumentation astronomique. Deux solutions principales ont alors émergé. La première, proposée par Christiaan Huygens fut de diminuer le rayon de courbure et l'épaisseur des lentilles utilisées afin de diminuer l'épaisseur de verre à traverser et donc l'importance des aberrations chromatiques. Cette solution présente cependant l'inconvénient de

18. Descartes, 1668 , p. 65.

19. Verdet, 1990, p. 162.

20. Watson, 2004, p. 91-92.

21. C'est d'ailleurs ce même principe que Newton utilisera pour étudier la décomposition de la lumière par un prisme. 
conduire à une augmentation de la distance focale des lunettes et à des tubes toujours plus grands. En effet, la distance focale est d'autant plus grande que le rayon de courbure est faible (déviation des rayons lumineux moins importante) et de plus celle-ci varie comme le carré du diamètre de l'objectif.

Or, les astronomes ont toujours besoin d'augmenter ce diamètre d'ouverture - les Anglo-Saxons parlent à ce titre d'aperture fever ${ }^{22}$. En effet, l'ouverture d'un télescope, mesurée par la taille du diamètre de son miroir primaire, est dépositaire des objectifs scientifiques de la mission. L'augmentation du diamètre permet de recueillir plus de lumière et donc de détecter des sources plus faibles, d'où une augmentation de la quantité d'observations réalisées. En outre, le pouvoir de résolution augmente aussi, ce qui se traduit par l'augmentation de la qualité des observations. Dans leur quête de données à exploiter, les scientifiques ont donc tendance à construire - ou faire construire - des télescopes toujours plus imposants. Cette tendance au gigantisme est dès lors motivée par des facteurs externes au sens où ceux-ci dérivent d'un souci de performance utilitaire et non de nécessités techniques, et ces nouveaux géants vont d'ailleurs rapidement se heurter à un certain nombre de contraintes techniques.

Le double objectif de (1) diminuer la courbure des lentilles utilisées et (2) augmenter le diamètre de l'objectif a amené les lunettes à bientôt dépasser les 30 puis les 40 mètres de longueur jusqu'à atteindre 46 mètres en 1641. Ayant besoin d'échafaudages, de longs mâts ou grues pour les maintenir, ces télescopes étaient très peu maniables et surtout très fragiles : la moindre vibration, le moindre coup de vent risquait de détruire complètement le dispositif. Ces lunettes à très grande focale sont, pour reprendre le vocabulaire de la TRIZ ${ }^{23}$, la manifestation d'une contradiction technique typique des phases de saturation ${ }^{24}$ : il s'agit d'un conflit entre deux effets, qui ne peuvent être obtenus en même temps car toute tentative d'améliorer l'un conduit à détériorer l'autre ${ }^{25}$. L'amélioration d'une fonction utile conduit

22. Watson, 2004, p. 10.

23. La TRIZ, acronyme russe de "théorie de résolution des problèmes inventifs " est une théorie, mais aussi une méthode d'analyse et de résolution, des problèmes techniques mise au point par l’ingénieur soviétique Genrich Altshuller dans les années 1950.

24. Altshuller, 1999.

25. Analogue technique d'un optimum de Pareto au sens d'" allocation des ressources pour laquelle il n'existe pas une alternative dans laquelle tous les acteurs seraient dans une meilleure position ». 
à amplifier un effet néfaste, ou inversement, la réduction d'un effet néfaste s'accompagne d'une réduction d'une fonction utile. Dans le cas présent, l'augmentation de la distance focale permet de diminuer les aberrations chromatiques mais augmente la fragilité de l'objet technique : il y a donc contradiction entre la performance du dispositif et sa robustesse.

Une solution originale, bien qu'anecdotique, au problème de l'hypertrophie des tubes des lunettes fut proposée en 1675 par les frères Huygens qui proposèrent de se passer complètement de tube : ainsi naquit la sous-lignée des "télescopes aériens" (tubeless aerial telescope $e^{26}$ ). L'objectif était monté à l'intérieur d'un petit tube de fer fixé sur une rotule pivotante elle-même disposée sur un mât réglable, tandis que l'oculaire était monté dans un autre tube, les deux tubes étant maintenus alignés par une corde.

Cette idée, aussi élégante soit-elle, ne constitue toutefois pas une solution pérenne pour la lignée des télescopes réfracteurs dans la mesure où les problèmes de fragilité et de maniabilité demeurent : la contradiction principale n'étant pas dépassée, il serait plus judicieux de parler d'une tentative de contournement, d'un détour ${ }^{27}$, plus que d'une résolution du problème technique. Les télescopes réfracteurs à très long tube ainsi que les télescopes aériens peuvent être qualifiés de dispositifs hypertéliques, à la fois par analogie avec la biologie ${ }^{28}$ et au sens de Simondon ${ }^{29}$. En effet, l'allongement de la distance focale de l'instrument pour diminuer la courbure des lentilles afin de diminuer - et non supprimer - les aberrations chromatiques relève d'une logique de conception trop dépendante du donné technique, immédiatement saisissable par les concepteurs, pour constituer un véritable progrès technique. Elle donne plutôt l'illusion d'un progrès par l'amélioration des performances rendue possible par l'hypertrophie de certaines caractéristiques (la distance focale) au détriment d'une réflexion sur une éventuelle évolution du fonctionnement du dispositif technique.

La deuxième solution au problème des aberrations chromatiques relève d'une autre logique de conception et conduit à la mise au point de la lunette achromatique par Chester Moore Hall en $1729^{30}$. Lidée est de

26. King, 2011, p. 54.

27. Simondon, 2008, p. 139.

28. « Développement exagéré d'un organe qui peut aller jusqu'à compromettre la vie de l'individu ». 29. Pour Simondon, un dispositif technique est qualifié d'hypertélique quand son fonctionnement implique une suradaptation à un usage et un milieu particulier.

30. King, 2011, p. 144. 
revenir au principe de fonctionnement de la lentille - la réfraction - et de s'en servir pour contrecarrer les problèmes liés au caractère dispersif du verre. Une lentille achromatique est une lentille composée de deux types de verre de dispersion différente. Le premier élément est une lentille convexe en verre crown, de dispersion relativement faible, tandis que le second est une lentille concave en verre fint, de dispersion plus élevée. Les deux lentilles sont disposées l'une à côté de l'autre et conçues de sorte que la dispersion chromatique de l'une soit compensée par celle de l'autre. La conjonction des deux forme un doublet achromatique permettant de focaliser deux longueurs d'onde différentes au même foyer et d'ainsi réduire drastiquement les aberrations.

Breveté en 1758 par l'opticien anglais John Dollond (déclenchant une querelle de priorité avec Chester Moore Hall), le doublet achromatique va se généraliser dans la conception des lunettes ${ }^{31}$. Une évolution ultérieure de cette logique de conception fut la mise au point, dès 1765, du triplet apochromatique permettant de réaliser des lunettes dites apochromatiques. Lidée fut d'utiliser non plus deux mais trois éléments afin de focaliser trois longueurs d'onde différentes au même foyer, contribuant à une nette diminution des aberrations chromatiques. Enfin, le développement du verre en fluorite dans les années 1960, a permis de combiner quatre éléments au sein de lunettes superachromatiques, permettant de focaliser quatre longueurs d'onde au même point et améliorant d'autant la qualité des images produites. Notons qu'ici le progrès n'a plus seulement lieu à l'échelle de l'individu (la lunette) mais aux échelles élémentaire (lentilles) et même infra-objectale (type de verre).

La meilleure compréhension scientifique des principes sous-jacents et la volonté de contrôler le schème technique sont à l'origine de progrès au sein de la lignée qui, à la différence des évolutions hypertéliques, n’engendrent pas de contradictions. Le passage de la lunette classique à la lunette achromatique puis apochromatique et enfin superachromatique traduit une logique d'évolution différente de la tendance au gigantisme : la complexification correctrice, qui désigne les modifications structurales par l'ajout ou la complexification d'éléments permettant de corriger les défauts de l'objet. Les deux logiques peuvent néanmoins se combiner. L'invention du doublet achromatique et de ses successeurs a rendu à nouveau possible

31. Grillot, 1986, p. 280. 
l'emploi de lentilles plus épaisses, de distance focale moins importante, et donc de réduire la taille des instruments de plusieurs ordres de grandeur. Les évolutions ultérieures ont relancé la course au gigantisme : à partir du début du XIX ${ }^{e}$ siècle, la collaboration entre le verrier Pierre-Louis Guinand et l'instrumentaliste Joseph von Fraunhofer donne naissance à l'ère des " grands réfracteurs ${ }^{32}$ ". La découverte par le premier de nouveaux procédés de fabrication du verre Flint permit au second de réaliser une lunette achromatique de 24 centimètres d'ouverture pour une distance focale de " seulement " 4 mètres (à comparer avec la lunette d'Hevelius qui possédait une ouverture de 12 centimètres pour une focale de 46 mètres). Au cours du XIX ${ }^{\mathrm{e}}$ siècle, le diamètre de ces grandes lunettes va plus que quadrupler, passant de 24 centimètres à 102 centimètres pour la lunette de l'observatoire Yerkes et culminant à 125 centimètres pour la lunette de démonstration de l'Exposition universelle de Paris $1900^{33}$.

Cette lignée des grands réfracteurs finit aussi par rencontrer des contradictions techniques insurmontables, de véritables blocages technologiques. Tout d'abord, comme dans la lignée des réfracteurs non achromatiques, l'allongement du tube finit par être problématique. L'emploi de lentilles plus larges permit certes de réduire la distance focale mais au prix d'une augmentation du poids de la structure : le seul tube de la lunette de Yerkes pèse plus de 75 tonnes ce qui impacte grandement la maniabilité du dispositif. En outre, l'utilisation de lentilles de grande taille présente, intrinsèquement, deux problèmes majeurs. Le premier réside dans la difficulté à usiner des verres homogènes d'un tel volume conduisant à l'impossibilité d'obtenir des lentilles de bonne qualité de plus d'un mètre. Le second problème provient quant à lui des déformations des lentilles dues à la gravité - on parle de lens sagging - une lentille ne pouvant être supportée que par les bords, un poids trop important provoque une distorsion de la forme de la lentille qui ne focalise plus les rayons de la bonne façon. Cette nouvelle contradiction technique entre la taille et la qualité de l'objectif est impossible à résoudre car elle découle du principe même de la réfraction lumineuse ; elle entraîne une saturation définitive de la lignée des télescopes

32. Robert, 1967 , p. 344-350.

33. Celle-ci reste du point de vue scientifique plutôt anecdotique dans la mesure où, figée en position horizontale, elle ne permettait que des observations limitées. Elle sera d'ailleurs démontée à la fin de l'exposition, faisant de l'observatoire de Yerkes la plus grande lunette officiellement en activité. 
réfracteurs du fait de l'existence d'une limite objective du diamètre d'ouverture des lunettes (autour du mètre). La lignée des réfracteurs, saturée, allait être bientôt supplantée par la lignée concurrente des réflecteurs ${ }^{34}$.

\section{Concurrence des télescopes réflecteurs et substitution}

Proposé dès 1636 par Marin Mersenne ${ }^{35}$, théorisé en 1663 par James Grégory et réalisé en 1668 par Isaac Newton, le télescope réflecteur va peu à peu se substituer au réfracteur pour devenir l'instrument majeur de l'astronomie. Si les aberrations chromatiques sont un problème inhérent à l'usage de lentilles et au phénomène de réfraction, les télescopes réflecteurs recourant à des miroirs et basés sur le principe de réflexion lumineuse en sont quant à eux purement et simplement dénués ${ }^{36}$. C'est ce constat qui poussa Newton à abandonner les télescopes réfracteurs et à concevoir, en 1668, le premier télescope à base de miroirs. Composé d'un miroir primaire concave sphérique fait d'un alliage d'étain et de cuivre, et d'un miroir secondaire plan et oblique caractéristique des télescopes de "type Newton ", ce télescope présentait l'avantage d'être dénué d'aberrations chromatiques et d'être particulièrement compact. Deux autres types de design existent pour les télescopes composés de miroirs : le type grégorien ${ }^{37}$ théorisé en 1663 et le type Cassegrain ${ }^{38}$ mis au point en 1672.

Cependant, la difficulté d'usiner des miroirs de la forme désirée est responsable d'aberrations géométriques qui limitent les diamètres d'ouverture par rapport à la lignée concurrente des réfracteurs. Ces limitations industrielles ont été des freins à la diffusion et à l'épanouissement de cette lignée. Les premiers télescopes réflecteurs utilisaient des miroirs métalliques, en bronze tout d'abord puis en speculum ${ }^{39}$. S'ils présentaient l'avantage d'être dénués d'aberrations chromatiques, ils possédaient l'inconvénient de ternir à l'usage (oxydation due à l'atmosphère) et possédaient un faible pouvoir

34. Watson, 2004, p. 181.

35. Ariotti, 1975, p. 302-321.

36. Newton, 1955.

37. Miroir primaire concave hyperbolique, miroir secondaire concave elliptique.

38. Miroir primaire concave parabolique, miroir secondaire convexe hyperbolique.

39. Alliage métallique composé de deux tiers de cuivre et d'un tiers d'étain, et formant une surface hautement réfléchissante une fois poli. 
réfléchissant (un tiers de la lumière incidente étant perdue). Il fallait donc les polir relativement souvent, ce qui, à la longue, contribuait à éloigner les miroirs de leur forme optimale et donc à dégrader la qualité du télescope.

Pour ces raisons, les télescopes réfracteurs, notamment achromatiques, furent longtemps préférés aux réflecteurs. Il fallut plusieurs décennies avant que ces derniers ne s'imposent dans la communauté des astronomes : "quelques télescopes furent fabriqués à partir de 1720, [...] il fallut attendre qu'Edward Scarlett trouve la manière de réaliser de bons miroirs pour que la fabrication prit quelques extensions ${ }^{40}$ ". Puis, en 1856, Karl August von Steinheil et Léon Foucault adaptèrent indépendamment le procédé Liebig d'argenture du verre, permettant de déposer une couche d'argent sur un miroir en verre ${ }^{41}$. Cette couche d'argent était non seulement beaucoup plus réfléchissante et plus durable que la surface des miroirs métalliques, mais elle avait aussi l'avantage de pouvoir être enlevée et redéposée sans changer la forme du substrat de verre. Ce nouveau procédé industriel réellement inventif rendait possible la réalisation de miroirs de grande taille et d'une qualité suffisante pour que la lignée des télescopes réflecteurs manifeste ses potentialités et vienne se substituer à la lignée des réfracteurs jusque-là dominante. Le début du $\mathrm{xx}^{\mathrm{e}}$ siècle voit la construction de nombreux observatoires modernes, conçus pour l'imagerie photographique de précision et installés sur des sites particuliers ${ }^{42}$ (éloignés des villes, en altitude). Par la suite, la lignée des télescopes réflecteurs concentre l'essentiel des efforts de développement et connaître nombre d'évolutions non seulement selon la tendance au gigantisme mais aussi selon la tendance de complexification correctrice. Si les télescopes réflecteurs sont dénués d'aberrations chromatiques, ils possèdent en revanche un certain nombre d'aberrations géométriques. C'est la meilleure compréhension scientifique de ces aberrations et de leurs causes qui constitue le moteur du progrès de la lignée des réflecteurs : tout comme les progrès de la dioptrique avaient permis l'évolution des lentilles et des lunettes, une meilleure compréhension des phénomènes physiques mis en jeu va induire l'évolution des miroirs et des télescopes réflecteurs.

40. Daumas, 2003, p. 67.

41. King, 2011, p. 262.

42. Grillot, 1986, p. 275 : «c'est au cours du $19^{\mathrm{e}}$ siècle [...] qu’ont été créés les grands observatoires français autres que celui de Paris ». 
Le télescope Ritchey-Chrétien (RCT), inventé en 1910, constitue à ce titre une avancée majeure au sein de la lignée ${ }^{43}$. Un télescope constitué de miroirs sphériques souffrira toujours d'aberrations sphériques. Si les miroirs sont rendus paraboliques pour corriger l'aberration sphérique, ils sont affligés alors de coma (déformation de l'image qui semble avoir une queue (coma) comme une comète) et d'astigmatisme (lignes verticales et horizontales sont focalisées à des distances différentes). Avec deux miroirs hyperboliques, le Ritchey-Chrétien élimine à la fois les aberrations sphériques et le coma jusqu'à l'ordre 3. Cela ouvre à un plus grand champ de vision utile même si de tels télescopes souffrent encore d'aberrations aux ordres supérieurs (coma d'ordre 5 et astigmatisme). Lajout d'un troisième miroir en 1935 permettra de remédier à ce problème et donnera naissance au Three Mirror Anastigmat (TMA) qui, comme son nom l'indique, en plus des aberrations d'ordre inférieur, est dénué d'astigmatisme. Parallèlement à ces développements, l'invention de la lame de Schmidt puis de la chambre de Schmidt en 1930 (par l'opticien allemand Bernhard Schmidt) donne naissance à une nouvelle lignée : la lignée des télescopes catadioptriques. Une lame de Schmidt (Schmidt corrector plate) est une lentille asphérique conçue pour que, une fois combinée avec un miroir sphérique, elle supprime le coma tout en compensant l'astigmatisme du réflecteur.

Le développement du RCT puis du TMA tout comme l'invention de la lame de Schmidt relèvent de deux logiques de conception différentes pour corriger les aberrations de coma et d'astigmatisme. Si la première solution consiste essentiellement en des modifications structurales, la seconde introduit un nouvel élément. Simondon nomme recrutement ${ }^{44}$ cet ajout d'un élément au sein de l'individu au cours du processus de concrétisation. Cela correspond aussi à l'hybridation des opérations de réfraction et de réflexion pour former un nouveau schème technique, celui de la lignée des télescopes catadioptriques. L'évolution est analogue à celle rencontrée lors de l'étude des réfracteurs et relève de la même logique de complexification correctrice. La lignée des télescopes catadioptriques va à son tour connaître une bifurcation selon que le correcteur employé occupe l'intégralité de l'ouverture (full aperture corrector) ou n'occupe qu'une portion choisie de celle-ci (sub aperture corrector). Dans les deux cas, l'utilisation d'éléments dioptriques permet de corriger les aberrations, constituant ainsi

43. Bely, 2003.

44. Simondon, 2008, p. 146. 
un progrès sur le plan phénoménotechnique ${ }^{45}$. Néanmoins, elle se heurte à nouveau aux limitations liées à l'impossibilité d'usiner de grandes lentilles : par conséquent, si le télescope de Schmidt-Cassegrain est l'un des plus populaires parmi les astronomes amateurs, il ne peut constituer le cœur opérationnel des grands observatoires. Au sein de ces derniers, ce sont les Ritchey-Chrétien et les TMA qui sont majoritairement employés et qui constituent, à ce jour, les dernières évolutions d'architecture du télescope ${ }^{46}$. Tout comme dans la lignée des lunettes, les efforts de développement ont ensuite porté sur les échelles infra et supra individuelles.

On l'a vu, la tendance à l'augmentation du diamètre d'ouverture - la taille du miroir primaire vu comme élément technique - est apparue dès les premiers télescopes et demeure dans toutes les lignées. Cependant, d'un point de vue mécanologique, elle n'est que seconde par rapport à la problématique de réduction des aberrations qui a conduit à la diversification des schèmes et à leur complexification à l'intérieur de chaque lignée. Bien que ces lignées aient coexisté pendant une longue période, les télescopes basés sur des miroirs possèdent intrinsèquement certains avantages par rapport à ceux utilisant des lentilles. À l'absence d'aberrations chromatiques s'ajoute la possibilité industrielle de pouvoir usiner des miroirs plus grands, les lentilles étant limitées à des diamètres d'un mètre tout au plus. Le passage des lentilles aux miroirs constitue ainsi la première innovation de rupture dans l'évolution au niveau élémentaire, permettant un gain en fidélité tout en ouvrant tout un champ de potentialités en termes de sensibilité. Les deux évolutions majeures ultérieures de la lignée des télescopes réflecteurs joueront d'ailleurs sur ces deux dimensions : le passage des miroirs en speculum à ceux en aluminium puis à support en verre permettront un gain du coefficient de réflexion tout en étant plus facile à usiner et autorisant de plus grandes tailles ${ }^{47}$. On retrouve les logiques sous-tendant l'évolution des lunettes. D’un côté une tendance au gigantisme (Fig. 1), essentiellement motivée par des causes externes, de l'autre, une complexification croissante du dispositif (passage de deux à trois miroirs, utilisation de miroirs

45. Bachelard, 1934, p. 17 : "Dès qu'on passe de l'observation à l'expe®rimentation [...] il faut que le phénomène soit trié, filtré, épuré, coulé dans le moule des instruments, produit sur le plan des instruments [...]. La véritable phénome囚nologie scientifique est donc bien essentiellement une phénoménotechnique. »

46. Zirker, 2005, p. 317.

47. Grillot, 1986, p. 281. 
hyperboliques plutôt que sphériques, ajout d'un correcteur) résultant de nécessités techniques internes.

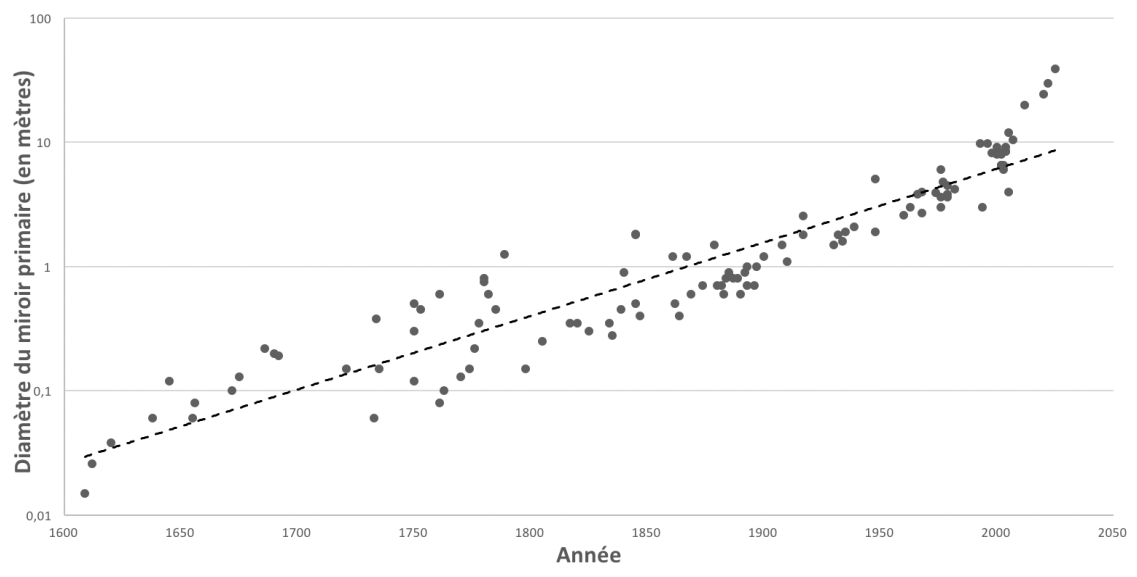

Fig. 1. - Évolution du diamètre d'ouverture des télescopes optiques, illustrant la course au gigantisme

Sources des données : Racine, 2004, p. 77-83 ; King, 2011 ; Watson, 2004.

\section{Graphique des auteurs}

Les miroirs à support de verre ont progressé jusqu’à atteindre une taille de 5-6 mètres ${ }^{48}$. Au-delà, leur masse fait qu'ils se déforment sous leur propre poids et qu'il devient impossible de maintenir leur forme idéale. On retombe donc sur les problèmes de sagging conduisant à un retour des aberrations géométriques dont on aurait pu penser s'être débarrassé. Objets plus concrets au sens de Simondon ${ }^{49}$, les télescopes réflecteurs se heurtaient à une contradiction technique face à laquelle plusieurs solutions s'offraient ${ }^{50}$ : alléger la masse du miroir ou corriger en temps réel sa forme. La première solution agit sur les éléments déjà existants et passe par le développement de nouveaux types de miroirs (miroirs fins), de nouvelles structures de miroir (nid d'abeilles) ou de nouveaux matériaux ( $\mathrm{SiC}$, CFRP). La seconde passe par l'ajout de nouveaux éléments (les actuateurs) et le développement d'une nouvelle technique : l'optique active. Les

48. Le télescope de Hale, mis en service en 1949, et le BTA-6 de 1975, sont, avec respectivement cinq et six mètres d'ouverture, les plus grands télescopes à support de verre classiques.

49. Simondon, 2012, p. 56 : «L'objet technique concret, c'est à dire évolué [...], tend vers la cohérence interne ".

50. Cheng, 2009, p. 102-120. 
deux recherches convergent puisque les miroirs plus légers nécessitent le recours à l'optique active pour ne pas plier sous leur propre poids.

La logique de conception de l'optique active s'est prolongée en direction de l'utilisation de miroir segmenté ${ }^{51}$. L'idée est de réaliser un grand miroir à partir de segments plus petits et juxtaposés ; l'alignement devant être de l'ordre de la longueur d'onde du rayonnement incident. Cette précision nanométrique n’a été possible que grâce aux développements de l'informatique. Parallèlement, l'idée d'utiliser non plus plusieurs segments pour réaliser un plus grand miroir mais plusieurs miroirs pour réaliser l'équivalent d'un plus grand télescope est apparue. L'apparition des télescopes à miroirs multiples a là encore été rendue possible par les progrès en traitement du signal et notamment la possibilité d'utiliser l'interférométrie aux longueurs d'onde visibles. Enfin, la technologie des miroirs fins a progressé de façon à donner naissance aux miroirs ultra-fins, utilisés notamment en optique adaptative.

Optique active et miroir segmenté ainsi qu'optique adaptative et interférométrie sont le prolongement aux autres échelles des deux logiques de 114 progrès identifiées au niveau de l'individu télescope, à savoir la tendance au gigantisme et la complexification correctrice.

\section{Évolutionnisme et génétique technique}

Les objets techniques évoluent au sein d'une lignée par concrétisation selon Simondon, c'est-à-dire vers une plus grande synergie interne ${ }^{52}$. L'épuisement des potentialités d'évolution d'une génération d'objets techniques conduit à des évolutions mineures concernant les matériaux ou la structure. Une fois l'objet saturé, celui-ci ne peut progresser à nouveau qu'en changeant totalement son plan d'organisation. De même que dans la TRIZ, l'évolution technique se retrouve sur un " point selle ", c'est-àdire dans une situation où progresser dans une direction implique une dégradation dans une autre ${ }^{53}$. La notion de contradiction technique pro-

51. Wilson, 2003.

52. Simondon, 2012, p. 26.

53. Cavallucci, 2001, p. 115-120 : «Loi n 5 (développement inégal des parties) : Les parties d’un système évoluent à des rythmes inégaux. Plus le développement des pièces est inégal, plus le système devient complexe. Il en résulte des contradictions physiques et techniques qui, à leur tour, créeront 
posée par Genrich Altshuller rejoint donc les analyses de Simondon sur le processus de saturation des objets techniques : les solutions fondées sur un trade-off correspondent à une stagnation. Une véritable invention technique implique un dépassement de la contradiction, car " c'est dans les incompatibilités naissant de la saturation progressive du système de sous-ensembles que réside le jeu des limites dont le franchissement constitue un progrès ${ }^{54}$ ".

Dans les lignées étudiées ici, le passage d'une génération d'objets techniques à la suivante s'accompagne de ce processus de concrétisation : c'est le cas en particulier du passage des premières lunettes aux lunettes achromatiques ou encore le passage du RCT au TMA. En effet, les différentes lentilles se compensent pour éliminer les erreurs résiduelles liées au caractère dispersif des verres employés tout comme les différents miroirs travaillent de concert pour supprimer les aberrations dues à l'asphéricité des optiques mobilisées : ce qui était obstacle devient moyen de réalisation ${ }^{55}$. Le processus de concrétisation peut alors être vu comme le résultat de la dialectique existante entre les deux logiques de conception identifiées précédemment. La complexification correctrice tend à augmenter le nombre de sous-systèmes de l'objet technique, et donc la probabilité de rencontrer des " incompatibilités ", quand la tendance au gigantisme tend à saturer l'objet selon une certaine direction. Arrive un point où l'objet technique se retrouve sur un point selle qu'il ne peut franchir qu'en se réinventant, en se concrétisant.

Dans son célèbre article de $1935^{56}$, Lucien Febvre indiquait les trois dimensions dans lesquelles devait se déployer toute tentative d'histoire des techniques : faire l' " histoire technique de la technique », étudier l' "insertion de l'invention technique dans la série des faits scientifiques » et rattacher cette activité au sein "des autres activités humaines ". Même si notre méthodologie répond avant tout à la première exigence, ce sont les trois dimensions que la mécanologie génétique tente de concilier à travers l'étude des objets techniques : la compréhension des tendances techniques réclame

\footnotetext{
des problèmes pour le développement futur du système. Le système ne peut alors se développer logiquement que si ces contradictions sont résolues ".

54. Simondon, 2012, p. 32.

55. Altschuller, 1999, p. 32: «We can compensate for the evil, and extract something useful from it instead of eliminating it ".

56. Febvre, 1935.
} 
celle des phénomènes physiques qui sous-tendent les fonctionnements et les contradictions de l'objet ; les logiques de conception doivent aussi être éclairées par les dynamiques sociales qui affectent les concepteurs. En tant qu'instrument scientifique, le télescope est sans doute moins soumis aux surdéterminations économiques et socioculturelles qui pèsent sur les objets d'usage $^{57}$. Avec son histoire largement documentée et s'étendant sur plus de quatre siècles, l'astronomie peut donner lieu à une étude mécanologique telle que la nôtre, pouvant se coupler à une histoire sociologique de l'institutionnalisation des pratiques et de la discipline.

La prise en compte des facteurs socio-économiques, des progrès et des besoins scientifiques, ainsi que des possibilités technologiques développées dans d'autres domaines sont autant de prolongements naturels de notre démarche : la mobilisation du concept de lignées techniques ne permet donc pas seulement d'exhiber la « logique interne ${ }^{58}$ " d'évolution des dispositifs télescopiques mais aussi des pistes d'articulation avec les autres dimensions de l'histoire des techniques. En réinterprétant le processus de concrétisation à l'œuvre au sein de ces lignées, non plus comme la réponse à une "nécessité interne $e^{59}$ " mais comme émergent d'une dialectique entre deux tendances d'évolution (et deux logiques de conception), nous visons par cette démarche à réconcilier les pratiques internalistes et externalistes de l'histoire des techniques au profit d'une véritable " génétique technique ${ }^{60} "$.

\section{Bibliographie}

Altshuller Genrich, The Innovation Algorithm. TRIZ, Systematic Innovation and Technical Creativity, Technical Innovation Center, Worcester, 1999.

Ariottr Piero E., "Bonaventura Cavalieri, Marin Mersenne, and the Reflecting Telescope", ISIS, vol. 66, n 3, 1975, p. 302-321.

BaChelard Gaston, Le Nouvel Esprit scientifique, Presses universitaires de France, Paris, 1934.

Bewy Pierre (dir.), The Design and Construction of Large Optical Telescopes, Springer, New York, 2003.

57. Simondon, 2012, p. 31 : contrairement à l'automobile par exemple, "objet technique chargé d'inférences psychiques et sociales [qui] ne convient pas au progrès technique ".

58. Maunoury, p. 56.

59. Simondon, 2012, p. 27.

60. Cotte, 2003, p. 201-213. 
Cavallucci Denis, "Integrating Altshuller's development laws for technical systems into the design process", CIRP Annals - Manufacturing Technology, vol. 50, 2001, p. 115-120.

Cheng Jingquan, The Principles of Astronomical Telescope Design, Springer, New York, 2009.

Cotтe Michel, "La génétique technique a-t-elle un avenir comme méthode de l'histoire des sciences ", dans REy Anne-Lise (dir.), Méthode et histoire. Quelle histoire font les historiens des sciences et des techniques?, Classique Garnier, Paris, 2003, p. 201-213.

Couderc Paul, Histoire de l'astronomie classique, Presses universitaires de France,

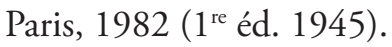

Daumas Maurice, Les Instruments scientifiques aux XVII et XVIII siècles, Éditions Jacques Gabay, Paris, 2003 (1 $1^{\text {re }}$ éd. 1953).

Descartes Renée, Discours de la méthode pour bien conduire sa raison et chercher la vérité dans les sciences, Édition Angot (Source numérisée : BnF/Gallica), Paris, 1668.

Febvre Lucien, "Réflexions sur l'histoire des techniques ", Annales d'histoire économique et sociale, $\mathrm{n}^{\circ} 36,1935$, p. 531-535.

Galilei Galiléo, Le Messager des étoiles, Seuil, Paris, 1992 ( $1^{\text {re }}$ éd. 1610).

Grillot Solange, "Les Instruments des observatoires français au XIX ${ }^{\mathrm{e}}$ siècle ", L'Astronomie, vol. 100, 1986, p. 275-289.

Khun Thomas, La Structure des révolutions scientifiques, Flammarion, Paris, 2008

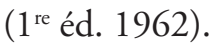

KIng Henry C., The History of the Telescope, Dover Publications, Buckinghamshire, 2011.

Koyré Alexandre, Du Monde clos à l'univers infini, Presses universitaires de France,

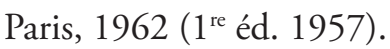

Koyré Alexandre, La Révolution astronomique, Les Belles Lettres, Paris, 2016

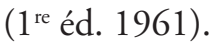

Lafitte Jacques, Réflexions sur la Science des Machines, Bloud \& Gay, Paris, 1932.

Maunoury Jean-Louis, La Genèse des innovations. La Création technique dans l'activité de la firme, Presses universitaires de France, Paris, 1968.

Newton Isaac, Traité d'optique, Gauthier-Villars, Paris, 1955 (1 ${ }^{\mathrm{re}}$ éd. 1704).

Racine René, "The Historical Growth of Telescope Aperture", Publications of the Astronomical Society of the Pacific, vol. 116, $\mathrm{n}^{\circ} 815,2004$, p. 77-83.

Roвert Waaland J., "Fraunhofer and the Great Dorpat Refractor", American Journal of Physics, vol. 35, n 4 1967, p. 344-350.

SÉRIs Jean-Pierre, La Technique, Presses universitaires de France, Paris, 2013.

Simondon Gilbert, Imagination et Invention (1965-1966), Les éditions de la Transparence, Chatou, 2008. 
Simondon Gilbert, Du Mode d'existence des objets techniques, Aubier, Paris, 2012

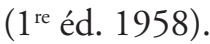

Simondon Gilbert, "Psychosociologie de la technicité ", dans Sur la Technique, Presses universitaires de France, Paris, 2014, p. 27-73.

Van Helden Albert, "The Invention of the Telescope", Transactions of the American Philosophical Society, vol. 67, n 4, 1977, p. 1-67.

Van Helden Albert, Dupré Sven, Van Gent Rob, Zuidervaart Huib (dir.), The Origins of the Telescope, Knaw Press, Amsterdam, 2010.

Verdet Jean-Pierre, Une Histoire de l'astronomie, Seuil, Paris, 1990.

Walker Christopher, Astronomy Before the Telescope, British Museum Press, Londres, 1999.

Watson Fred, Stargazer. The Life and Times of the Telescope, Da Capo Press, Cambridge, 2004.

Wilson Raymond, Reflecting Telescope Optics, Springer, New York, 2003 (1 ${ }^{\text {re }}$ éd. 1996).

Zirker Jack B., An Acre of Glass: a History and Forecast of the Telescope, Johns Hopkins University Press, Baltimore, 2005.

\section{Les auteurs}

Thomas Guy est ingénieur et actuellement doctorant en histoire et philosophie des techniques au Laboratoire de Recherche sur les Sciences de la Matière (CEALARSIM). Ses recherches portent sur le développement d'une méthode génétique et son application à l'histoire des instruments scientifiques. Il a notamment coécrit « L'étude des lignées phénoménotechniques. De Bachelard à Simondon et aux micromegas » avec Vincent Bontems dans le cadre de l'ouvrage collectif IL senso della tecnica. Saggi su Bachelard (Esculapio, 2017).Contact : thomas.guy@ cea.fr

Vincent Bontems est philosophe, chercheur au Laboratoire de Recherche sur les Sciences de la Matière (CEA-LARSIM) et chercheur associé au Centre de Gestion Scientifique (CGS-Mines Paris Tech). Il a récemment publié avec Vincent Minier et al., Inventing a Space Mission, the History of the Herschel Space Observatory, Springer, Cham, 2017.Contact : vincent.bontems@cea.fr 